\title{
Antiaging therapy: a prospective hypothesis
}

Pubse journal:

Drug Design, Development and Therapy

28 January 2015

Number of times this article has been viewed

\author{
Mohammad Rashid \\ Shahidi Bonjar' \\ Leyla Shahidi Bonjar ${ }^{2}$ \\ 'School of Dentistry, Kerman \\ University of Medical Sciences, \\ Kerman Iran; ${ }^{2}$ Department of \\ Pharmacology, College of Pharmacy, \\ Kerman University of Medical \\ Sciences, Kerman, Iran
}

Abstract: This hypothesis proposes a new prospective approach to slow the aging process in older humans. The hypothesis could lead to developing new treatments for age-related illnesses and help humans to live longer. This hypothesis has no previous documentation in scientific media and has no protocol. Scientists have presented evidence that systemic aging is influenced by peculiar molecules in the blood. Researchers at Albert Einstein College of Medicine, New York, and Harvard University in Cambridge discovered elevated titer of aging-related molecules (ARMs) in blood, which trigger cascade of aging process in mice; they also indicated that the process can be reduced or even reversed. By inhibiting the production of ARMs, they could reduce age-related cognitive and physical declines. The present hypothesis offers a new approach to translate these findings into medical treatment: extracorporeal adjustment of ARMs would lead to slower rates of aging. A prospective "antiaging blood filtration column" (AABFC) is a nanotechnological device that would fulfill the central role in this approach. An AABFC would set a near-youth homeostatic titer of ARMs in the blood. In this regard, the AABFC immobilizes ARMs from the blood while blood passes through the column. The AABFC harbors antibodies against ARMs. ARM antibodies would be conjugated irreversibly to ARMs on contact surfaces of the reaction platforms inside the AABFC till near-youth homeostasis is attained. The treatment is performed with the aid of a blood-circulating pump. Similar to a renal dialysis machine, blood would circulate from the body to the AABFC and from there back to the body in a closed circuit until ARMs were sufficiently depleted from the blood. The optimal application criteria, such as human age for implementation, frequency of treatments, dosage, ideal homeostasis, and similar concerns, should be revealed by appropriate investigations. If AABFC technology undergoes practical evaluations and gains approval, it would hold future promises such as: 1) prolonged lifespans; 2) slowed age-related illnesses such as low bone mass, weak muscular systems, diabetes, arthritis, Alzheimer's disease, and impaired memory in the elderly; 3 ) reduced health expenses; 4) reduced cosmetic surgeries performed on the elderly; 5) healthier astronauts in extended outer space journeys; 6) reduced financial burden of advanced care for the elderly imposed upon both government and society; and 7) rejuvenating effects in healthy, non-aged individuals.

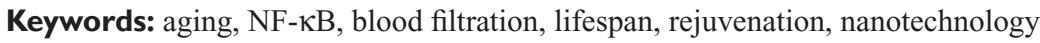

\section{Introduction}

Aging is a cascade of creeping functional deteriorations that occur throughout the body. In fact, researchers do not know enough about why humans age. One human desire is to retard aging from middle age onward. We would be also enchanted with rejuvenation innovations, if developed. Some outcomes of the major breakthrough research on triggers and retardation of aging are as follows. In 2009, Harrison et al from the Department of Aging and Geriatric Research, College of Medicine, Institute on Aging, University of Florida, Gainesville, Florida, reported that a drug slowed aging in middle-aged mice. ${ }^{1}$ The drug was rapamycin, a therapeutic used in humans to minimize rejection of transplanted organs. They noticed that it extended the lifespan of mice by up to $14 \%$. They also claimed that the drug worked even when given to older mice late in life. They pos-
Correspondence: Leyla Shahidi Bonjar Department of Pharmacology, College of Pharmacy, Kerman University of Medical Sciences, Kerman, 76169I3555, Iran Tel +989131985712

Fax +983432120725

Email shahidileylaa@gmail.com License The full terms of the License are available at http://creativecommons.org/licenses/lby-nc/3.0/. Non-commercial uses of the work are permitted without any further (c) (i) (-) 2015 Shahidi Bonjar and Shahidi Bonjar. This work is published by Dove Medical Press Limited, and licensed under Creative Commons Attribution - Non Commercial (unported, v3.0) permission from Dove Medical Press Limited, provided the work is properly attributed. Permissions beyond the scope of the License are administered by Dove Medical Press Limited. Information on
how to request permission may be found at: http://www.dovepress.com/permissions.php 
tulated that rapamycin may increase the lifespan by delaying death from maladies such as cancer, and/or by postponing mechanisms of aging. In 2010, Villeda et al at the Department of Neurology and Neurological Sciences in the School of Medicine, Stanford University, Stanford, CA, found that decline in neurogenesis and cognitive impairments in mice are partially correlated to variations in blood-borne factors. ${ }^{2}$ By using heterochronic parabiosis (circulation-sharing young/old mouse pairs), they showed that blood-borne factors can prevent or boost neurogenesis in adult mice in an age-dependent manner. They observed similar results by injecting cell-free plasma from older mice into younger mice and vice versa. This was a turning point in aging research, because they showed substances in the blood of young mice made older brains perform like younger brains, and the blood of older mice made younger brains perform like older brains. They observed a threefold increase in generation of new nerve cells in older mice receiving "younger" blood; while the younger members of old/young mice pairs presented a decrease in generation of new nerve cells receiving "older" blood. These scientists demonstrated that "eotaxin" plasma levels were correlated with neurogenesis in young/old mouse pairs. Elevated peripheral level of eotaxin in younger mice lowered adult neurogenesis and weakened learning and memory. In their experiments with humans, Villeda et $\mathrm{al}^{2}$ also performed a study on blood and cerebrospinal fluid samples taken from healthy people between the ages of 20 years and 90 years, and they demonstrated a parallel age-related increase of eotaxin. In this regard, they raised the question of whether it might be possible to shield the brain from aging by removing or relieving the effects of such apparently harmful blood-borne substances.

In 2013, Zhang et al at the Department of Molecular Pharmacology, Albert Einstein College of Medicine, Bronx, New York, demonstrated that the hypothalamus was critical for the progress of whole-body aging in mice, and the elemental basis involved hypothalamic immunity mediated by IкB kinase- $\beta$ (IKK- $\beta)$, nuclear factor $\kappa \mathrm{B}(\mathrm{NF}-\kappa \mathrm{B})$, and associated microglia. ${ }^{3}$ Interestingly, they developed few interventional models to show retardation of aging. Their experiment indicated that extension of lifespan was accessible by inhibition of aging-related activation of IKK- $\beta$ and NF- $\kappa B$ in the hypothalamus. Further, they revealed that IKK- $\beta$ and NF- $\mathrm{KB}$ inhibit gonadotropin-releasing hormone (GnRH). Their evaluations revealed that GnRH decelerated aging, and that GnRH treatment improved aging-impaired neurogenesis in mice. They concluded two main points: 1) the hypothalamus had a definite programmatic role in aging development and 2) immune prevention or GnRH revival in the hypothalamus/brain would offer two possible tactics for improving lifespan and would fight aging-related health maladies. Zhang et $\mathrm{al}^{3}$ also showed that NF-kB activation in neurons in the hypothalamus increased with age and accordingly concluded that hypothalamus activity affects cognitive and physical decline in mice. They proved that mice injected with a drug that inhibited the activity of immune cells (called microglia in the hypothalamus) performed better, and they revealed that less age-related decline in muscle strength, skin thickness, and bone mass occurred in the mice. The researchers also demonstrated that mice lived longer and that those mice who received NF- $\kappa \mathrm{B}$ stimulant showed more age-related decline and died earlier than normal. Their results were similar in both sexes.

In 2014, Katsimpardi et al at the Department of Stem Cell and Regenerative Biology, Harvard University, Cambridge, ${ }^{4}$ proved that "systemic factors" taken from younger mice could increase neurogenesis and improve olfactory discrimination in aging mice. They postulated that determination of such factors that retard age-dependent deterioration may highlight new procedures in treating agerelated neurodegenerative and neurovascular diseases. Also, age-related oxidative stress in the peripheral nervous system and its degenerative consequences have been investigated by several researchers. ${ }^{5-8}$ Loss of proper physiological function of neurons due to the accumulation of oxidative damage is documented based on "Oxidative Stress Theory of Aging". ${ }^{9}$ The buildup of age-related molecules, along with their inefficient removal by homeostatic mechanisms, deteriorates vulnerable peripheral nerves. ${ }^{10}$ To slow down age-related negative consequences, the present hypothesis is proposed to maintain systemic concentration of agerelated molecules close to youth homeostasis from middle age onward and in the elderly.

\section{The hypothesis}

In recent times, it has been revealed that inhibiting activities of several ARMs in blood decelerates aging in mice. If that scenario is also applicable for humans, it is clear that inhibiting or optimizing such molecular activities would increase lifespan and combat aging-related health problems. To translate these findings into medical treatment is the basic core of the present paper's prospective hypothesis.

Extracorporeal blocking of ARMs (eg, known aging factors as IKK $-\beta, N F-\kappa B$, and others that meticulous investigations would reveal in the future) would decelerate aging effects when performed from middle age onward; such techniques would be applicable in the elderly, too, but should not start early in life. The present hypothesis contains within it the means 
to suggest a method to adjust ARM titers in blood to nearyouth homeostasis. An "antiaging blood filtration column" (AABFC) is a proposed device that would immobilize ARMs from blood. The AABFC would be used in a similar manner as a dialysis unit in artificial kidney machines; however, instead of a dialysis membrane, it utilizes an impermeable biocompatible membrane impregnated with ARM antibodies (ARM-ABs) on it's both sides. ARMABs would conjugate with ARM antigens; hence, they would be trapped inside the column. The membrane with its ARM-ABs would have a vast surface area for direct contact with blood components. While blood circulates in the AABFC, stationary ARM-ABs would attract and immobilize ARMs from the blood. Like a dialysis machine, blood would circulate from the body to the AABFC and from the AABFC back to the body in a closed circuit until ARMs were sufficiently well removed from the blood. The AABFC (Figure 1) and its tubing would be single-use and would be appropriately disposed of after each treatment. Appropriate treatment duration would be revealed by simple serological evaluation of ARM titers in blood returning to the body. When ARM titers in blood reached near-youth homeostasis, such homeostasis would indicate the end of a treatment session. Optimized use of the AABFC would clear a person's blood from an excess of ARMs. This treatment may need to be performed three or four times a year, or as optimization studies should reveal.

\section{AABFC specifications}

The AABFC membrane would be a non-porous, organic biocompatible polymer with approximately $200 \mu \mathrm{m}$ thickness; it would be longitudinally folded and packed in the AABFC to have extended contact surfaces on both sides with the blood that would pass through it. The ARM-ABs would be engineered with the ability to reside firmly on both sides of the membrane to act as the retention platforms to trap ARMs from the blood stream. Approximate internal volume of the column could be approximately $100 \mathrm{~mL}$. Including connection tubes, at any given point of time, the total volume of blood undergoing processing would be approximately $200 \mathrm{~mL}$; however, the exact settings should be discovered through appropriate investigations.

\section{AABFC treatment}

AABFC treatment setup would be similar to devices reported by Shahidi Bonjar. ${ }^{11,12}$ Figure 1 shows the proposed treatment setup, indicative of the path of the blood from and back into the body. Aseptically, blood passes through the AABFC with the aid of a peristaltic blood pump. Pressure monitors, heparin injections, and air traps would serve in the line to provide safe and smooth circulation. Before blood enters the column and after leaving it, blood samples would be taken at certain intervals to detect ARM titers with the aid of serological assays. All tubing and the AABFC would be applied aseptically to prevent infection; however, they would be single-use and would be properly disposed of after treatment.

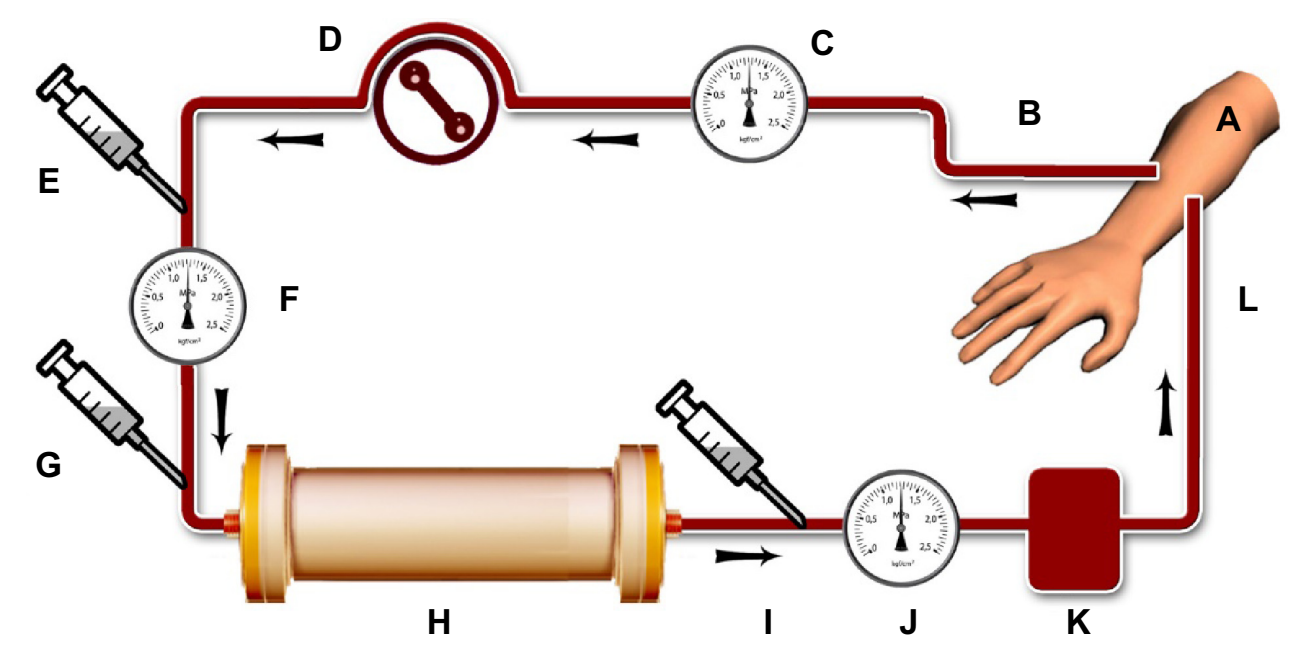

Figure I Schematic diagram of proposed treatment using "antiaging blood filtration column" (AABFC) for removing aging-related molecules (ARMs) from the blood stream of middle-aged and elderly people, a treatment that could reduce age-related physical declines in later life.

Notes: At each treatment session, the intent would be to set the ARM titer near to youth homeostasis. The AABFC would work aseptically with the aid of a blood pump in a closed circuit. Main components of the treatment would include: (A) the hand of a person to be treated; (B) blood removed from an artery through tubing; (C) an arterial pressure monitor; (D) a peristaltic blood pump; (E) a heparin pump (to prevent clotting); (F) an inflow pressure monitor; (G) an inflow sampling port to detect ARM titer before processing; and $(\mathbf{H})$ an AABFC, the central device to immobilize ARMs from blood. The AABFC bears a longitudinally folded biocompatible membrane impregnated with ARM antibodies (ARM-ABs) on both sides. ARM-ABs would conjugate with ARM antigens; hence, the ARM-ABs would be trapped inside the column. Components would also include (I) an outflow sampling port to detect ARM titer after processing; (J) a venous pressure monitor; (K) an air detector and air trap; and (L) processed blood returns back into the vein. All tubing and the AABFC would be applied aseptically; however, both tubing and the AABFC are single-use and would be properly disposed of after treatment. 


\section{Clinical significance}

Extracorporeal adjustment of ARMs would slow aging and alleviate aging-related health problems. The AABFC would immobilize quantities of ARMs from blood close to youth homeostatic values. The treatment is performed with the aid of a blood-circulating pump. Similar to a renal dialysis machine, blood would circulate from the body to the AABFC and from there back to the body in a closed circuit until ARMs were sufficiently depleted from the blood. The optimal application criteria, such as human age for implementation, frequency of treatments, dosage, ideal homeostasis, and similar concerns, should be delineated by appropriate investigations. If AABFC technology undergoes practical evaluations and gains approval, it would hold future promises such as: 1) prolonged lifespans; 2) slowed age-related illnesses such as low bone mass, weak muscular systems, diabetes, arthritis, Alzheimer's disease, and impaired memory in the elderly; 3) reduced health expenses; 4) reduced cosmetic surgeries performed on the elderly; 5) healthier astronauts in extended outer space journeys; 6) reduced financial burden of advanced care for the elderly imposed upon both government and society; and 7) rejuvenating effects in healthy, non-aged individuals.

\section{Probable side effects and remedies}

Similar to conventional hemodialysis, AABFC treatment may cause similar inconveniencies such as hypotension, muscle cramps, infection, clotting, itching, dry mouth, and anxiety. The cures are the same as those performed in hemodialysis; ${ }^{5}$ however, the problems would be much less in the AABFC than in patients with kidney failure taking frequent dialysis treatments.

\section{Why not use straight antibody injections?}

If ARM-ABs were injected straight into the blood stream, the immune system would be sensitized, and ARMs would be recognized as foreign antigens. Aside from probable allergic effects, production of anti-antibodies and phagocytosis by macrophages would counteract effectiveness of ARM-ABs. The efficacies would decrease more in subsequent injections because of elevated titer of anti-antibodies in the blood; however, extracorporeal removal of ARMs by the AABFC would not sensitize the immune system and would not have post-treatment adverse consequences.

\section{Conclusion}

AABFC treatment would be a targeted medical treatment. It could provide near-youth homeostasis of ARMs in the blood. Adjustment of ARMs in the blood from middle age and the elderly to close to youth homeostatic values may lead to the slowing down and alleviation of age-related illnesses. Reducing undesirable consequences of aging would enhance healthier lifespan, reduce aging-related health expenses, and improve quality of life; however, before its implementation in humans, meticulous investigations using animals is essential to prove its efficacy and safety. Details of the procedure and its use, such as appropriate starting age for implementation, frequency of applications, dosage, ideal homeostasis, and other similar concerns, should be clearly verified via appropriate future investigations.

\section{Dedication}

The authors would like to dedicate this article to those scientists whose efforts in the future may serve humanity by turning this hypothesis into reality.

\section{Disclosure}

The authors report no conflicts of interest in this work.

\section{References}

1. Harrison DE, Strong R, Sharp ZD, et al. Rapamycin fed late in life extends lifespan in genetically heterogeneous mice. Nature. 2009; 460(7253):392-395.

2. Villeda SA, Luo J, Mosher KI, et al. The ageing systemic milieu negatively regulates neurogenesis and cognitive function. Nature. 2011; 477(7362):90-94.

3. Zhang G, Li J, Purkayastha S, et al. Hypothalamic programming of systemic ageing involving IKK- $\beta$, NF- $\mathrm{KB}$ and GnRH. Nature. 2013; 497(7448):211-216.

4. Katsimpardi L, Litterman NK, Schein PA, et al. Vascular and neurogenic rejuvenation of the aging mouse brain by young systemic factors. Science. 2014;344(6184):630-634.

5. Goss JR, Stolz DB, Robinson AR, et al. Premature aging-related peripheral neuropathy in a mouse model of progeria. Mech Ageing Dev. 2011;132(8-9):437-442.

6. Agarwal S, Sohal RS. DNA oxidative damage and life expectancy in houseflies. Proc Natl Acad Sci U S A. 1994;91(25):12332-12335.

7. Blair IA. Lipid hydroperoxide-mediated DNA damage. Exp Gerontol. 2001;36(9):1473-1481.

8. Smith KJ, Kapoor R, Felts PA. Demyelination: the role of reactive oxygen and nitrogen species. Brain Pathol. 1999;9(1):69-92.

9. Bokov A, Chaudhuri A, Richardson A. The role of oxidative damage and stress in aging. Mech Ageing Dev. 2004;125(10-11):811-826.

10. Opalach K, Rangaraju S, Madorsky I, Leeuwenburgh C, Notterpek L. Lifelong Calorie Restriction Alleviates Age-Related Oxidative Damage in Peripheral Nerves. Rejuvenation Res. 2010;13(1):65-74.

11. Shahidi Bonjar L. "Nanogold detoxifying machine" to remove idle nanogold particles from blood stream of cancer patients treated with antibody-nanogold therapeutics. Med Hypotheses. 2013;80(5):601-605.

12. Shahidi Bonjar L. Design of a new therapy to treat snake envenomation. Drug Des Devel Ther. 2014;8:819-825. 


\section{Publish your work in this journal}

Drug Design, Development and Therapy is an international, peerreviewed open-access journal that spans the spectrum of drug design and development through to clinical applications. Clinical outcomes, patient safety, and programs for the development and effective, safe, and sustained use of medicines are a feature of the journal, which has also been accepted for indexing on PubMed Central. The manuscript management system is completely online and includes a very quick and fair peer-review system, which is all easy to use. Visit http://www.dovepress.com/testimonials.php to read real quotes from published authors.

Submit your manuscript here: http://www.dovepress.com/drug-design-development-and-therapy-journal 